\title{
Digital Lengthening with One Stage Autologous Bone Graft for Correction of Flail Toe: A case report
}

\author{
by Al Kline, DPM ${ }^{1} \otimes$, Sarah A. Curran, PhD, BSc(Hons) ${ }^{2}$,
}

The Foot and Ankle Online Journal 2 (12): 2

A flail toe, or sometimes called floppy toe is the result of aggressive resection of the proximal phalanx after hammertoe correction resulting in a toe that lacks stability and function. There is lack of function resulting from contracture of the toe and lack of toe purchase. There is usually associated pain from toe contracture that can press the toe against the top of closed shoes not to mention the poor cosmetic results causing embarrassment and pain. We present a case of flail toe corrected by autologous bone using graft from the shaft of the first metatarsal. After successful surgery, this one-stage lengthening procedure is stable and restored length to the toe.

Key Words: Flail toe, floppy toe, floating toe, autologous bone graft, one-stage toe lengthening.

Accepted: November, $2009 \quad$ Published: December, 2009

This is an Open Access article distributed under the terms of the Creative Commons Attribution License. It permits unrestricted use, distribution, and reproduction in any medium, provided the original work is properly cited. (The Foot and Ankle Online Journal (www.faoj.org)

$\mathrm{F}$ lail or floppy toe is described as a toe that has undergone too much bone resection after digital arthroplasty. This usually involves an aggressive resection to the head of the proximal phalanx involving a single toe. In a digital arthroplasty without fusion, the head of the proximal phalanx is usually resected at the surgical neck of the distal portion of the proximal phalanx. If the head, surgical neck and the shaft of the phalanx is resected to the base of the phalanx, a floppy or flail toe will result. It can also result from over-lengthening the extensor and flexor tendons ${ }^{1}$ of the digit or improper sequential release of the toe contracture.

Address correspondence to: Al Kline, DPM

3130 South Alameda, Corpus Christi, Texas 78404.

Email: alklinedpm@gmail.com.

${ }^{1}$ Adjunct Clinical Faculty, Temple University School of Podiatric Medicine, Barry University School of Podiatric Medicine. Private practice, Chief of Podiatry, Doctors Regional Medical Center. Corpus Christi, Texas, 78411.

${ }^{2}$ Senior Lecturer, Wales Centre for Podiatric Studies. University of Wales Institute, Cardiff, Western Avenue, Cardiff, CF5 2YB, United Kingdom.

\section{Case Report}

A 39 year-old female presented to the office with a floppy toe in March 2009. She complains of pain to the second toes and embarrassment while wearing closed shoes. She underwent aggressive arthroplasty to both second toes with bunionectomy almost 10 years prior to presentation. (Fig. 1) The toe is also floating' and would not purchase the ground. She also has a recurrent bunion deformity with pain and discomfort. She had gone to numerous foot surgeons who suggested a number of surgical corrective procedures including gradual callus distraction, syndactylization and even amputation. 


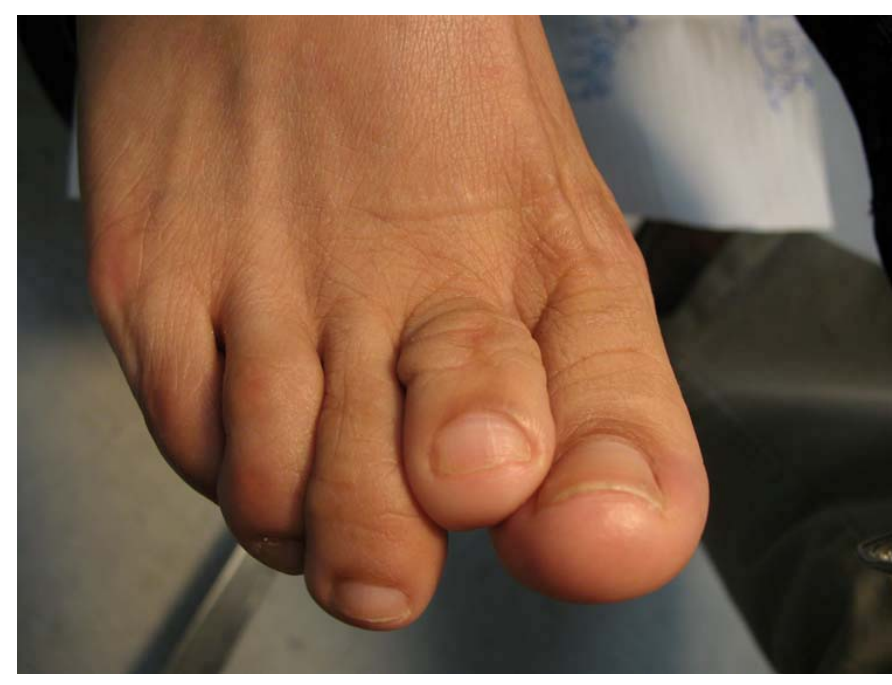

Figure 1 Initial presentation of severe flail toe 10 years post surgical arthroplasty.

She has tried Budin splinting and buddy-taping the toe without success. Radiographs revealed an overly aggressive resection of bone from the proximal phalanx. (Fig. 2) The toe also dorsiflexes and lacks plantarflexory purchase and 'rides' up on top of the foot on weight-bearing in a typical 'floating' toe fashion. (Fig. 3)

The patient has no allergies and is healthy without any medical problems. On physical examination, she has normal vascular and neurological status. On inspection, the toe is extremely floppy and is reducible on manual retraction without vascular compromise to the toe. (Fig. 4) Clinical evaluation of all the digits reveals hyperextensibility, flexibility and hypermobility to all the digits.

We discussed autologous one-stage lengthening of the toe that will be harvested from her first metatarsal while recorrecting her bunion deformity. We discussed the usual complications that may occur including failure of graft fusion, recurrent floppy toe and recurrent pain or deformity.

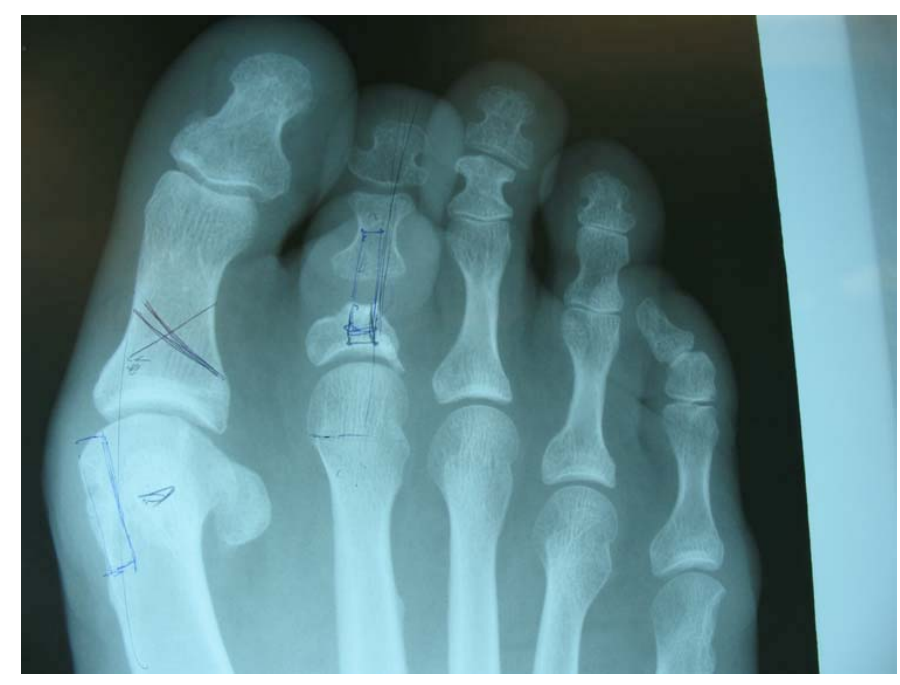

Figure 2 Pre-surgical planning of surgery. A bone graft will be removed from the first metatarsal and transposed to the second digit for lengthening. A revisional bunionectomy is also planned.

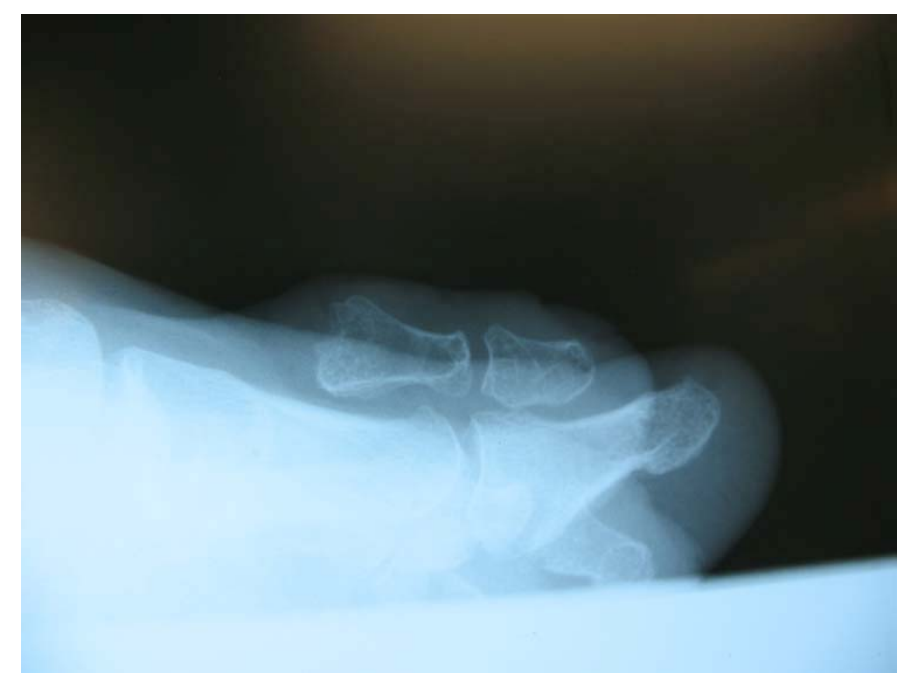

Figure 3 The lateral radiograph shows the flail toe in a floating orientation on weight-bearing view. This also represents as an iatrogenic floating toe. 


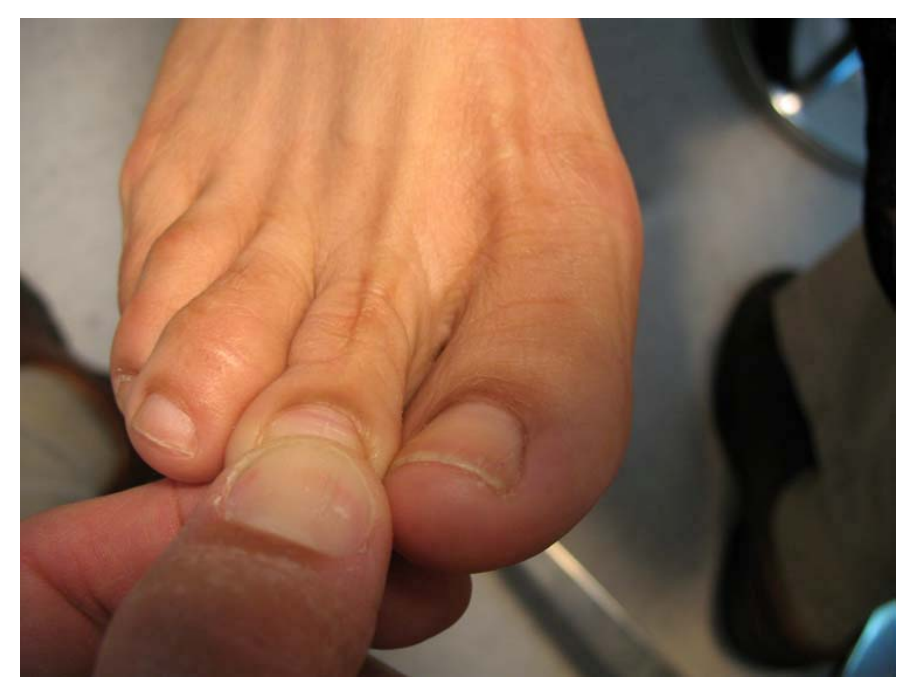

Figure 4 Initial presentation reveals good reducibility of the toe on distraction. Good refill is noted to the toe on stress distraction.

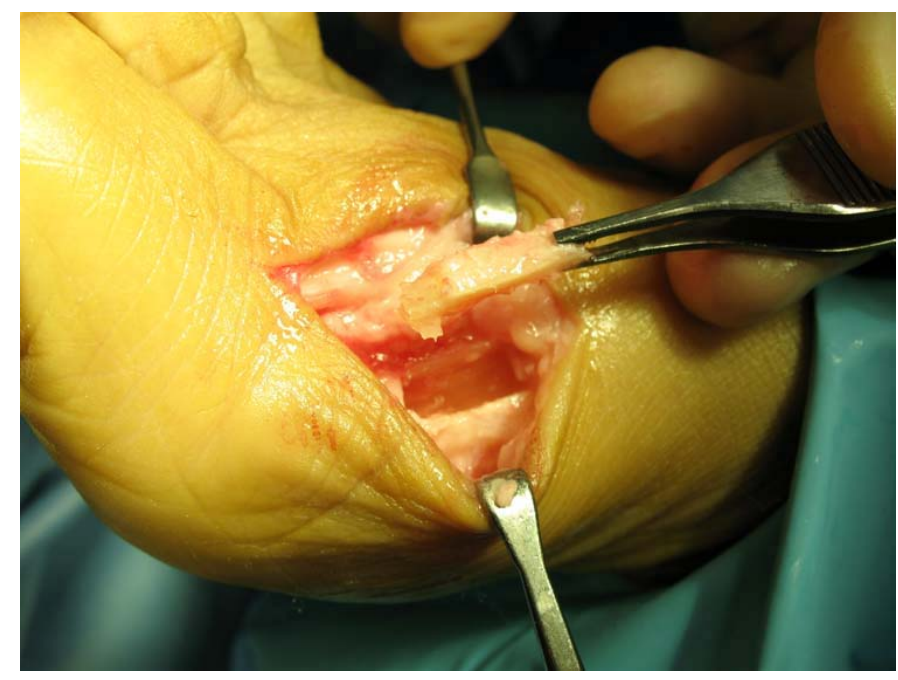

Figure 5 A cortical bone window is harvested from the first metatarsal to be used in the second toe lengthening procedure. The graft length was measured preoperatively on radiograph.

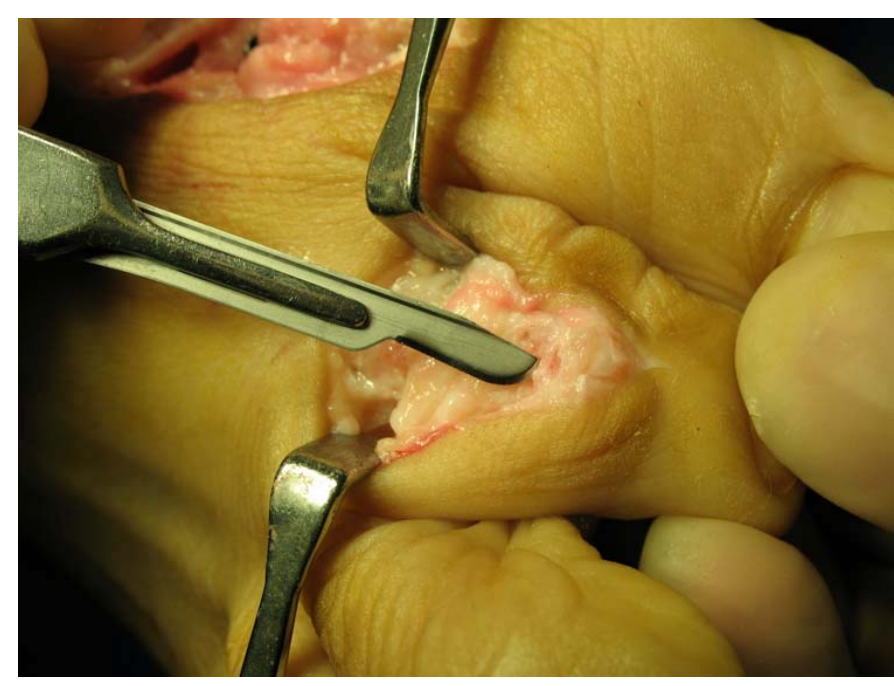

Figure 6 The middle phalanx is identified. It is important to strip the bone of all soft tissue scarring before surgical pinning of the bone graft.

\section{Surgical Correction}

The patient was scheduled for bunionectomy with bone graft harvest from the first metatarsal and autologous one-stage bone grafting of the second toe to promote anatomical lengthening. We estimated the graft length by measuring the amount of shortening of the entire toe in comparison to the third toe on radiograph. (Fig. 2) In surgery, two autologous bone struts from the medial eminence of the first metatarsal were harvested while performing the bunion and bone from a bone-window along the shaft of the metatarsal. An approximated $15 \mathrm{~mm}$ in length of autologous bone is harvested. (Fig. 5) The second toe is then opened dorsally along the proximal interphalangeal joint region. A significant amount of scarring is noted within the joint and resected carefully. To facilitate fusion of the graft the proximal stump of the phalanx and middle phalanx are sharply resected to expose bare bone (Fig. 6)

A simple smooth Kirschner wire is then drilled directly through the graft. (Fig. 7) The graft is then inserted within the second digital space while retracting the digit. (Fig. 8) 


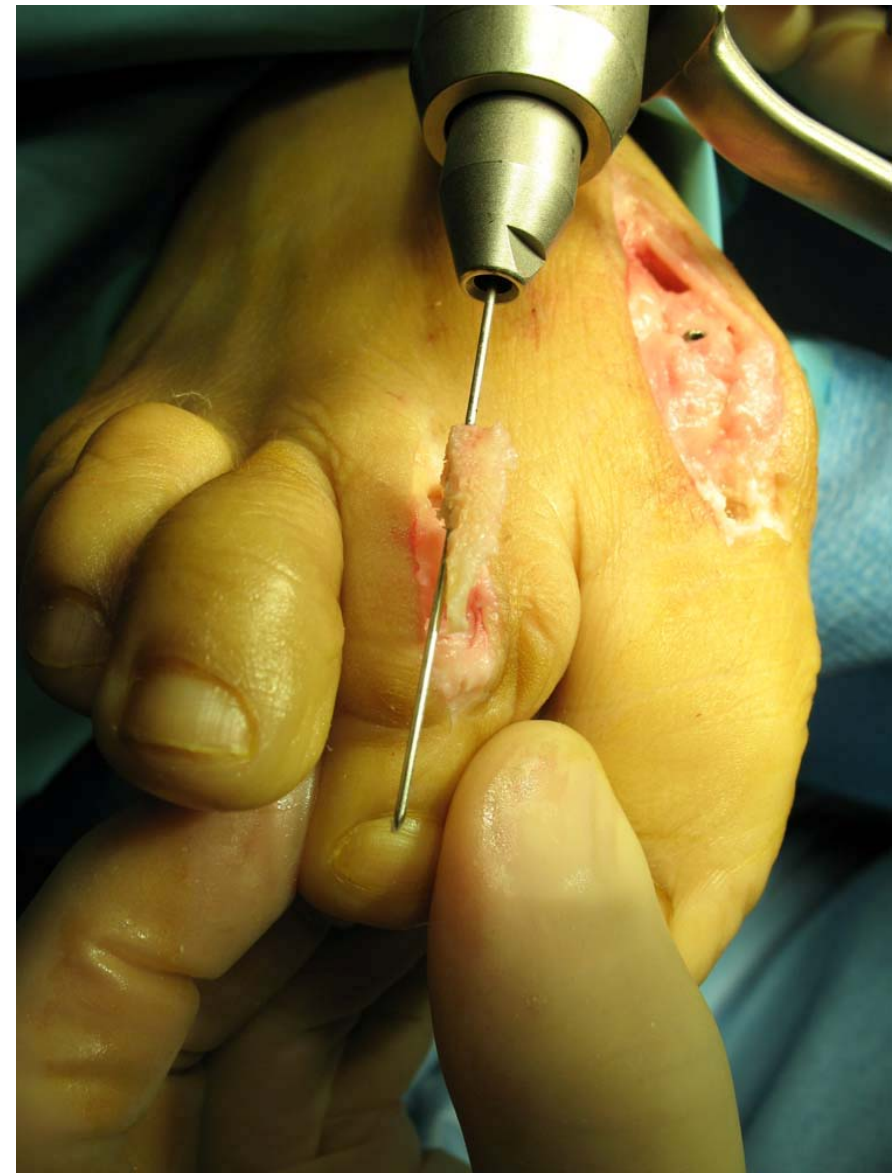

Figure 7 The bone graft is fixated with a smooth Kirschner wire and then retrograde pinned through the middle and distal phalanx out the end of the toe.

The pin is first directed distally along the distal toe then directed proximally along the proximal phalanx stump. The pin is then directly proximally into the metatarsal head to maintain digital stability in the postoperative period. (Fig. 9) The result can be visualized under radiographic intraoperative scan. (Figs. 10A and 10B) Three days after surgery, the pin and toe is seen in its anatomical alignment. (Fig. 11) Eight weeks after surgery, the graft has incorporated and the restoration of length to the toe has been restored. (Fig. 12)

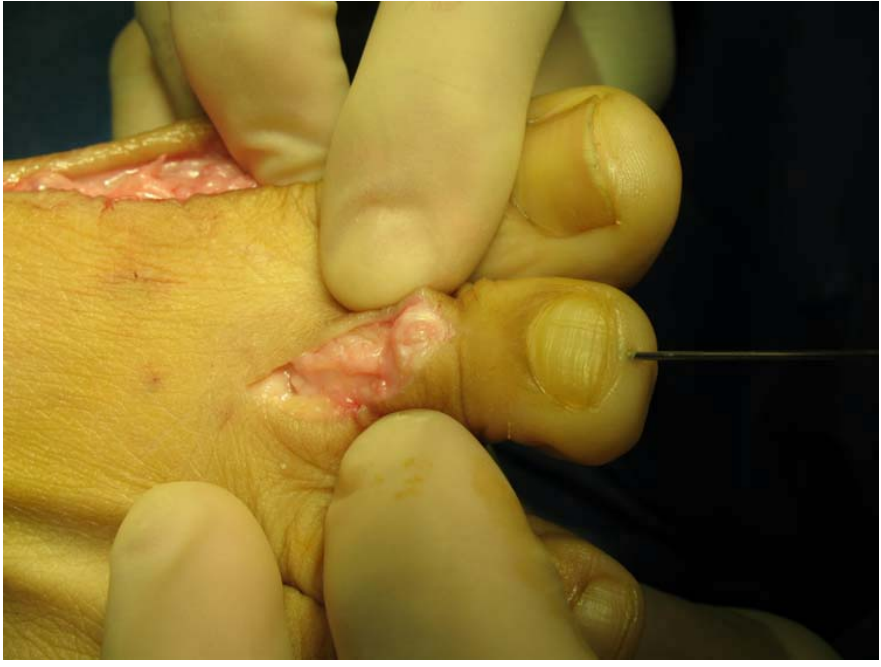

Figure 8 The bone graft is then fixated with a smooth Kirschner wire which is run through the proximal phalangeal stump after removing all soft tissue scarring.

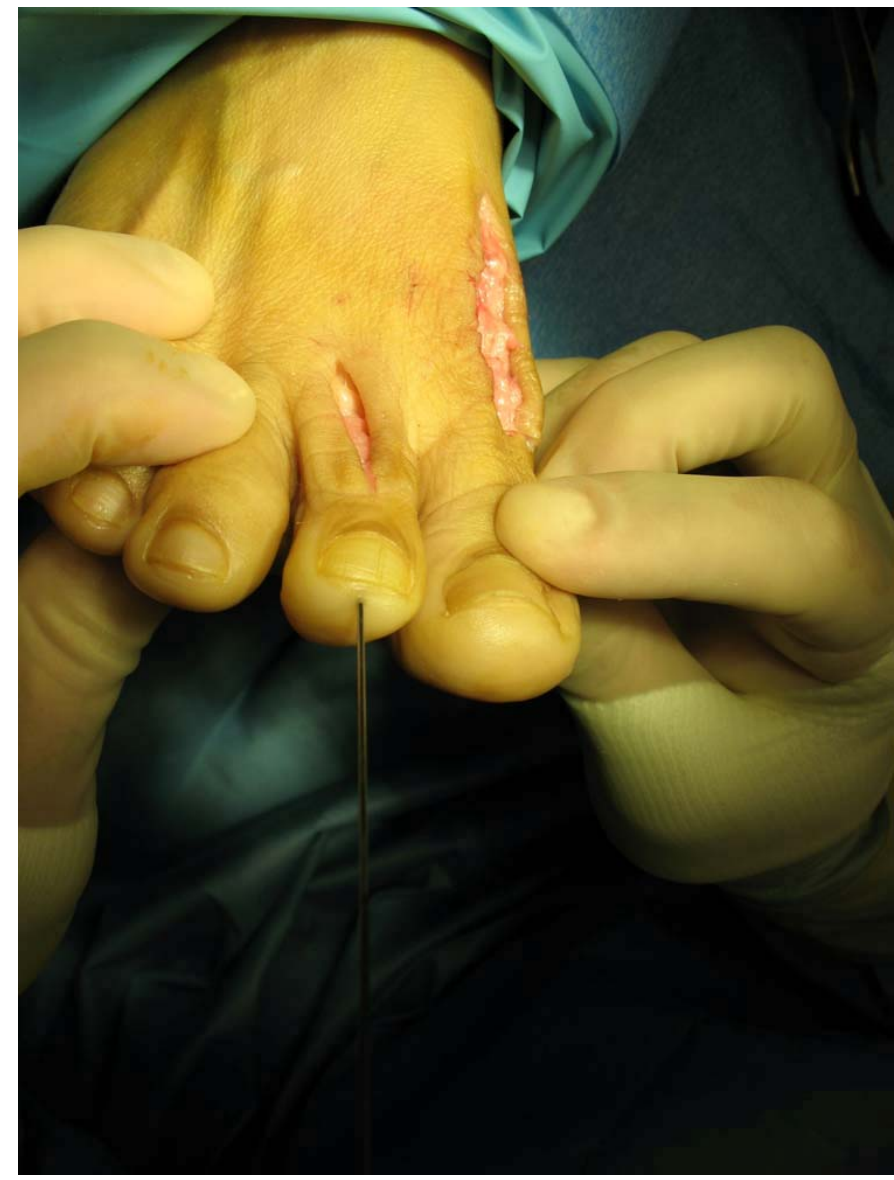

Figure 9 The pin is directed proximally through the metatarsal head to keep the toe from floating after extensor release. 


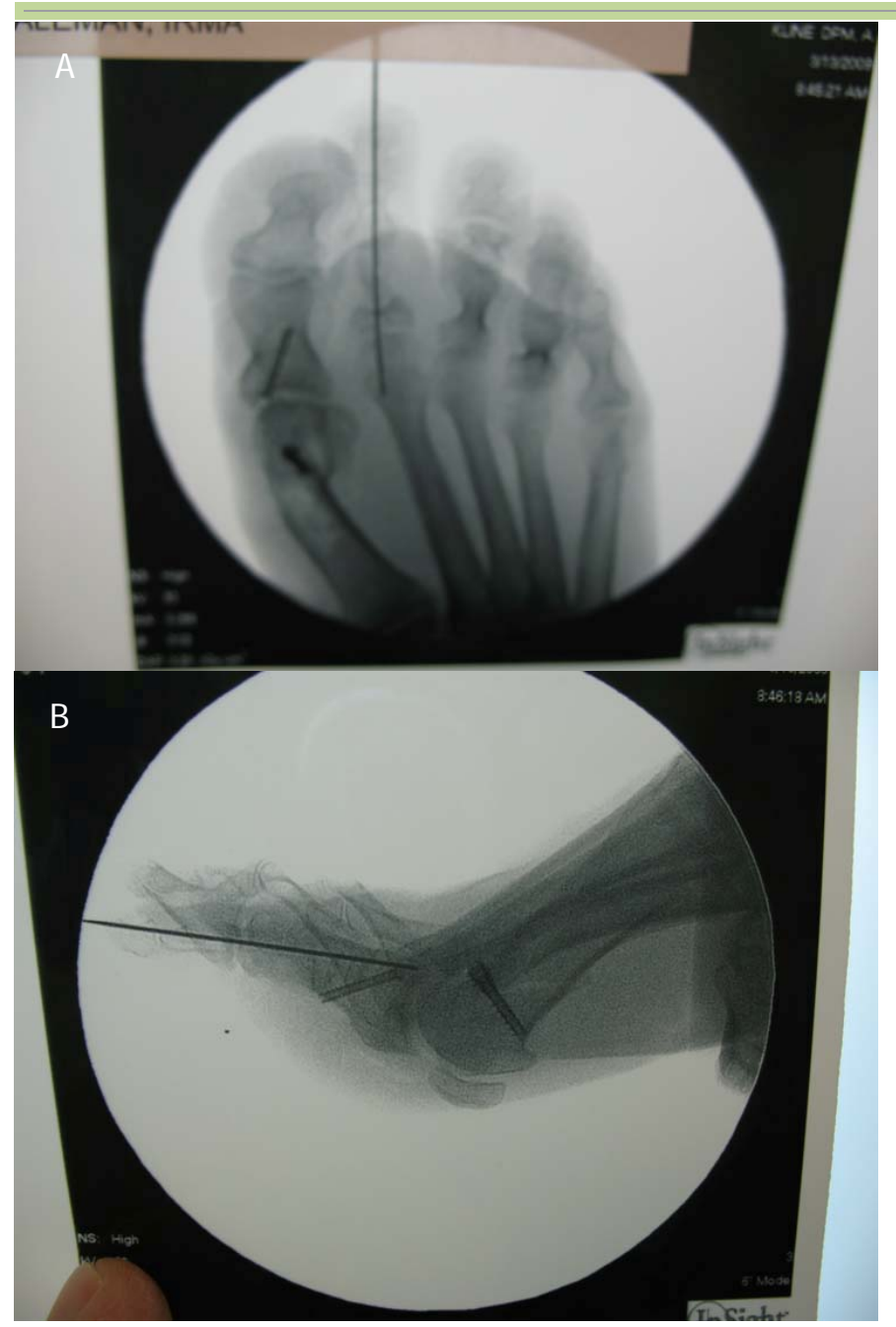

Figure 10A and 10B The revisional bunion and second digital bone graft is seen in proper anatomical orientation after fixation.

\section{Discussion}

In this report, the difference between 'floating toe' and 'flail toe' should be differentiated. A flail toe is surgeon induced by resecting too much bone from the phalanx, over-lengthening the digital extensor or flexor tendons and improper sequential release of a hammertoe contracture. ${ }^{1}$ A floating toe or floating toe syndrome, according to McGlamery, ${ }^{1,2}$, is where the toe lacks its structural integrity and fails to purchase the ground.

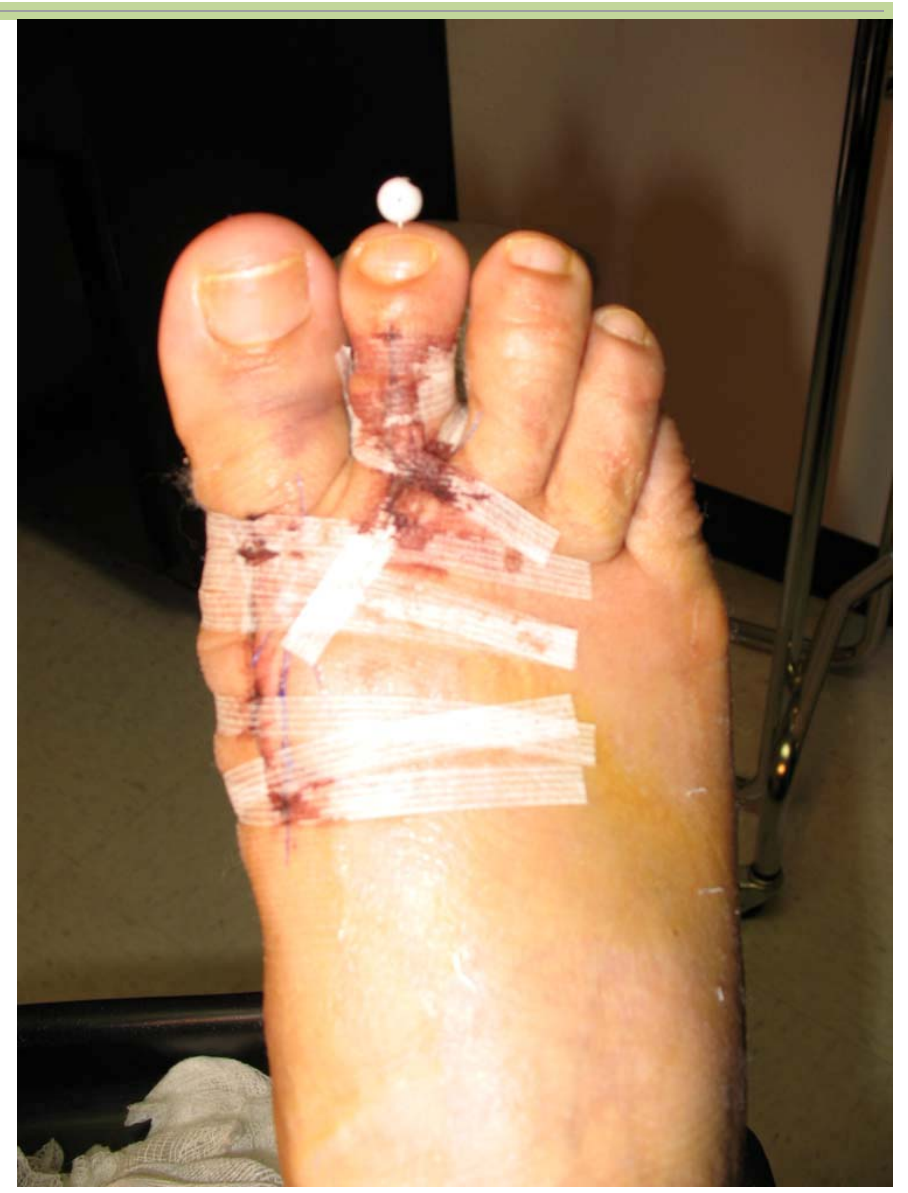

Figure 11 The foot 3 days after surgery.

This can be caused by a number of conditions that include both post surgical complications and anatomical deformities. This may include brachymetatarsia, surgically induced metatarsal head dorsiflexion or plantar or flexor plate disruption. Mann postulated that the syndrome is related to functional instability in the flexor mechanism caused by the inability to load the plantar fascia during weightbearing. ${ }^{1}$

Hammertoe correction is commonly corrected by two methods, 1) digital arthroplasty that includes resecting the head of the proximal phalanx and 2) by digital fusion after contractural release of the digit. This is commonly performed by intraoperative release of the proximal interphalangeal joint utilizing the Kelikian push-up test during contracture reduction. 


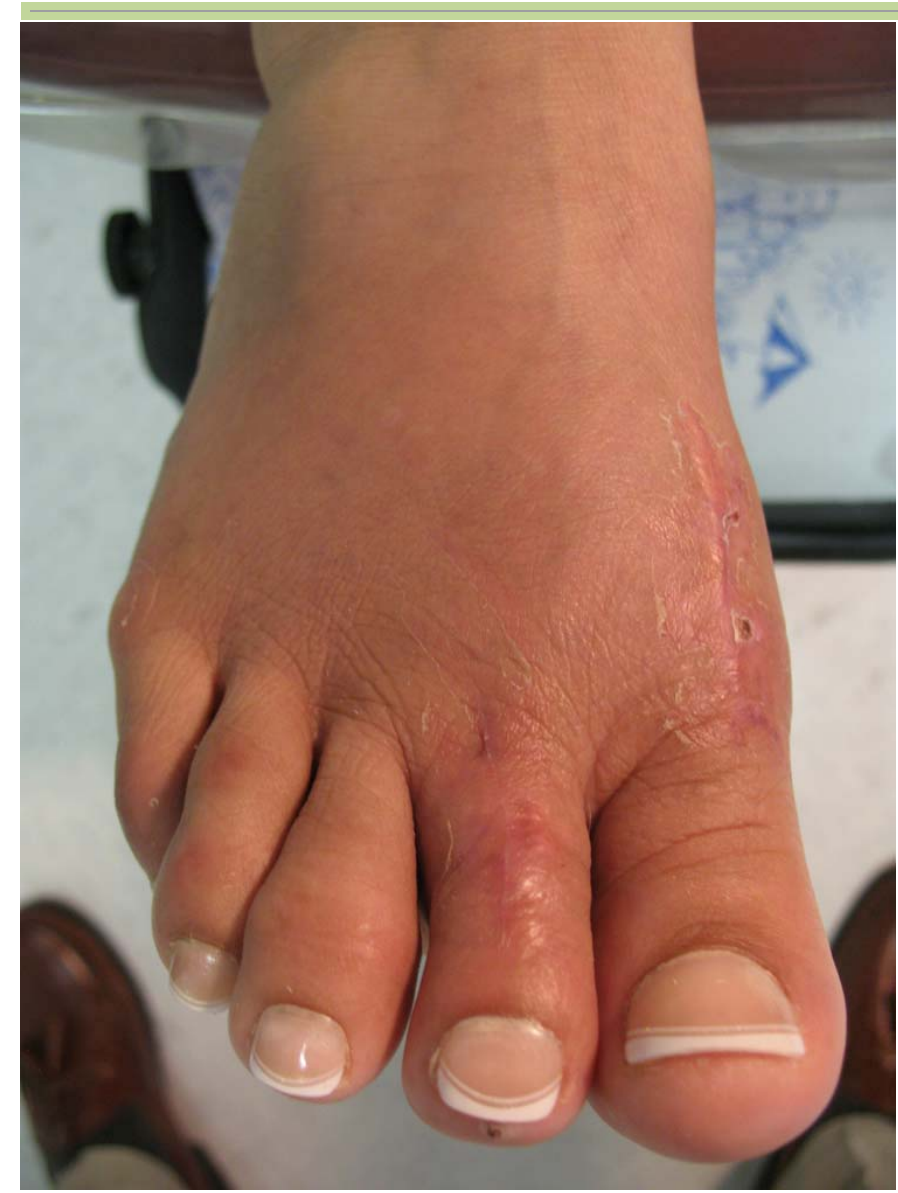

Figure 12 Eight weeks after surgery, the length of the toe has been restored.

Often the correction is performed by extensor tendon release, collateral ligament release to the head of the proximal phalanx, extensor hood resection and sometimes metatarsophalangeal joint release and decompression or Weil osteotomy. Hammertoes are also classified as either 'flexible' or 'rigid'. In our case, it was obvious that the flail toe was a direct result of too much bony resection after arthroplasty in an already flexible and reducible hammertoe. It is thus important to consider the physical examination and the flexibility of the toe before attempting an arthroplasty that may result in a floppy or flail toe. The author has also observed a number of cases of flail toe as a result of improper sequential release of the hammertoe.

(C) The Foot and Ankle Online Journal, 2009
As a result, the surgeon will often take too much bone in order to remove the hammertoe, but drastically shorten and destabilize the toe. Certainly, if the toes are already hyperflexible and hypermobile, even a less aggressive arthroplasty may result in a flail or floppy toe. Consideration of digital fusion may be more appropriate in digits that are hypermobile.

In this case, the only viable solution to a 'normal' appearing toe was to perform a digital lengthening using a bone graft. Bone grafting for metatarsal and digital lengthening have been shown to provide a safe and viable way to restore digital length after either iatrogenic shortening or genetic defects. A recent article by Lamm and Ades also described effective digital lengthening of a flail toe using an autologous bone graft in a patient with Raynaud's Disease. ${ }^{3}$ The overall success rate of autologous bone grafting for digital lengthening is high. In 1993, Mann, et al., reported an $82 \%$ success rate in a retrospective review of 22 cases in 13 patients who underwent onestage digital lengthening with autologous bone from the ipsilateral calcaneus. ${ }^{1}$

Most articles researched in the literature use the calcaneus or iliac crest as a source of bone for the autogenous graft. ${ }^{1,4,5}$ This appears to be the first reported correction of a flail toe using autologous bone graft from the first metatarsal. In our case, this was a convenience and pre-planned to avoid further graft sites. It appears that a well placed section of cortical bone from the first metatarsal is adequate to correct a flail toe deformity.

\section{References}

1. Mahan MS, Downey, MS, Weinfeld GD: Autogenous bone graft interpositional arthrodesis for the correction of flail toe. JAPMA 93(3): 167 - 173, 2003.

2. McGlamry ED: Floating toe syndrome. JAPA 72: 561 - 568, 1982.

3. Lamm BM, Ades JK: Gradual digital lengthening with autologous bone graft and external fixation for correction of flail toe in a patient with Raynaud's disease. JFAS, 48(4): 488vv494, 2009.

4. Mladick RA: Correction of hammer toe surgery deformity by Z-plasty and bone graft. Ann Plast Surg 4: 224, 1980

5. Laurie SW, Kaban LB, Mulliken JB, Murray JE: Donor-site morbidity after harvesting rib and iliac bone. Plast Reconstr Surg 73: 933, 1984.

6. Mahan KT: Calcaneal donor bone grafts. JAPMA 84: 1 - 9, 1994. 